Ferrata Storti Foundation

\title{
Hematopoietic stem and progenitor cells use podosomes to transcellularly cross the bone marrow endothelium
}

Haematologica 2020

Volume 105(12):2746-2756

\section{Correspondence:}

JAAP D. VAN BUUL

j.vanbuul@sanquin.nl

Received: May 4, 2018.

Accepted: January 20, 2020.

Pre-published: January 23, 2020.

doi:10.3324/haematol.2018.196329

(C)2020 Ferrata Storti Foundation

Material published in Haematologica is covered by copyright. All rights are reserved to the Ferrata Storti Foundation. Use of published material is allowed under the following terms and conditions:

https://creativecommons.org/licenses/by-nc/4.0/legalcode. Copies of published material are allowed for personal or internal use. Sharing published material for non-commercial purposes is subject to the following conditions:

https://creativecommons.org/licenses/by-nc/4.0/leǵalcode, sect. 3. Reproducing and sharing published material for commercial purposes is not allowed without permission in writing from the publisher.

\author{
Timo Rademakers,${ }^{1 *}$ Marieke Goedhart, ${ }^{2 \star}$ Mark Hoogenboezem, ${ }^{1}$ \\ Alexander García Ponce, ${ }^{3}$ Jos van Rijssel, ${ }^{1}$ Maryna Samus, ${ }^{4}$ Michael Schnoor, ${ }^{3}$ \\ Stefan Butz, ${ }^{4}$ Stephan Huveneers, ${ }^{5}$ Dietmar Vestweber, ${ }^{4}$ Martijn A. Nolte, ${ }^{2}$ \\ Carlijn Voermans ${ }^{2}$ and Jaap D. van Buul ${ }^{1}$
}

*TR and MG contributed equally as co-first authors

${ }^{1}$ Department of Plasma Proteins, Sanquin Research and Landsteiner Laboratory, Academic Medical Center, Amsterdam, the Netherlands; ${ }^{2}$ Department of Hematopoiesis, Sanquin Research and Landsteiner Laboratory, Academic Medical Center, Amsterdam, the Netherlands; ${ }^{3}$ Department of Molecular Biomedicine, Center of Research and Advanced Studies (CINVESTAV-IPN), Mexico-City, Mexico; ${ }^{4}$ Max Planck Institute for Molecular Biomedicine, Münster, Germany and ${ }^{5}$ Department of Medical Biochemistry, Academic Medical Center, Amsterdam, the Netherlands

\section{ABSTRACT}

B one marrow endothelium plays an important role in the homing of hematopoietic stem and progenitor cells (HSPC) upon transplantation, but surprisingly little is known on how the bone marrow (BM) endothelial cells regulate local permeability and hematopoietic stem and progenitor cells transmigration. We show that temporal loss of vascular endothelial-cadherin function promotes vascular permeability in BM, even upon low-dose irradiation. Loss of vascular endothelial-cadherin function also enhances homing of transplanted HSPC to the BM of irradiated mice although engraftment is not increased. Intriguingly, stabilizing junctional vascular endothelial-cadherin in vivo reduced BM permeability, but did not prevent HSPC cells migration into the BM, suggesting that HSPC use the transcellular migration route to enter BM. Indeed, using an in vitro migration assay, we show that human HSPC cells predominantly cross BM endothelium in a transcellular manner in homeostasis by inducing podosome-like structures. Taken together, vascular endothelial-cadherin is crucial for BM vascular homeostasis but dispensable for the homing of HSPC. These findings are important in the development of potential therapeutic targets to improve HSPC homing strategies.

\section{Introduction}

Hematopoietic stem cell transplantation is used to restore hematopoiesis in patients with (hematological) malignancies and disorders after chemotherapy and/or irradiation. The first step to therapeutic success of hematopoietic stem cell transplantation critically depends on the homing of sufficient numbers of hematopoietic stem- and progenitor cells (HSPC) to the bone marrow (BM). ${ }^{1}$ An important step in homing is the actual transmigration of re-infused HPSC across the $\mathrm{BM}$ endothelium to the underlying parenchyma. This extravasation event requires a set of specific molecular interactions that mediate the firm adhesion of the HSPC to the BM endothelial cells, and subsequent transmigration across the endothelial lining. ${ }^{2,3}$ For the diapedesis step, HSPC have to cross the endothelial barrier. It has been described that immune cells can cross the endothelial barrier on two different routes: the paracellular route, i.e., through the cell-cell junctions, or the transcellular pathway, i.e., through the cell body. ${ }^{4}$ It appears that neutrophils and monocytes prefer the paracellular route. However, T lymphocytes may have a preference for the transcellular route. ${ }^{5}$ Not only the immune cell type seems to matter here, but also the state of the endothelium: the brain endothelium allows more cells to traffic in a transcellular fashion, whereas in the lungs, all immune cells seem to prefer the junctions, i.e., the paracellular route. ${ }^{6,7}$ In addition to that, 
the amount of ICAM-1, one of the crucial adhesion molecules expressed by the inflamed endothelium can steer the route preference: overexpression of ICAM-1 drives lymphocytes towards the transcellular route. ${ }^{8-10} \mathrm{Up}$ to now, it is unclear why one immune cell prefers the paracellular route whereas the other crosses in a transcellular way.

Upon HPSC transplantation, it is desired to have as many HSPC to home to the bone marrow as possible. Understanding how these cells would cross the endothelium may open new opportunities to promote HPSC homing. It has been postulated that the route of the least resistance may play a role here. ${ }^{6}$ One important mediator of this hypothesis would be the barrier function of the endothelium. VE-cadherin is an important mediator of the endothelial barrier. ${ }^{11}$ It specifically controls the leakage in lung and skin tissues. In the BM, sinusoid lining appears to be continuous. ${ }^{12-14}$ This was demonstrated by scanning electron microscopy. The number of fenestrations found without the presence of a migrating immune cell was very limited. The role for VE-cadherin in the regulation of BM vascular integrity is not clear. As VE-cadherin is an important regulator for the barrier function as well as for transmigration, we investigated to what extend VE-cadherin regulates the integrity of the BM vasculature and the homing of HSPC in that respect.

Our data show that VE-cadherin regulates the vascular integrity in the BM upon low-dose irradiation conditions. Blocking of VE-cadherin with interfering antibodies increases permeability and promotes the homing of HPSC to the $B M$. Using the VE-cadherin- $\alpha$-catenin chimera knock-in animals, we could show that HPSC cross the BM endothelium in a transcellular manner, although basal and irradiation-induced permeability was reduced. Additional experiments implicate podosome structures on the HSPC to be involved in the transmigration event. Interestingly, blocking VE-cadherin did promote the number of HPSC that home to the BM. However, we did not find a beneficial effect for the long-term engraftment of these cells in the BM.

Together, we conclude that HSPC cross the BM endothelium in a transcellular fashion using podosomes. Temporal targeting of the BM-endothelial VE-cadherin will most likely result in a disturbance of the BM homeostasis and will not result in a faster recovery of the BM population upon irradiation. These findings are important in the development for potential therapeutic target to improve HSPC homing strategies.

\section{Methods}

\section{Mice}

The following strains were used: C57BL/6, C57BL/6-Ly5.1, VEcadherin/ $\alpha$-catenin7 and VE-cadherin-GFP.15 The VE-cadherin/ $\alpha$ catenin mice were characterized previously and showed no clear differences in hematopoiesis. ${ }^{16}$ Mice were maintained on a C57BL/ 6 background in the animal facilities of the Netherlands Cancer Institute (Amsterdam, the Netherlands) and the Max Planck Institute for Molecular Biomedicine (Münster, Germany) in specific pathogen-free conditions. All animal experiments were approved by the local Animal Ethical Committee in accordance with national regulations.

\section{Multi-photon imaging of vascular permeability}

Mice were injected with PBS containing GS- ${ }^{17,18}$ or VE-cadherin blocking or IgG1 isotype control antibody. ${ }^{19}$ See the Online Supplementary Material and Methods for further details.

\section{Murine HSPC homing assay}

See the Online Supplementary Material and Methods for details.

\section{Flow cytometry}

See the Online Supplementary Material and Methods for details.

\section{Confocal microscopy of murine BM sections}

See the Online Supplementary Material and Methods for details.

\section{Human HSPC migration assays}

Cord blood (CB) was collected according to the guidelines of Eurocord Nederland and CD34 $4^{+}$cells were isolated as previously described. ${ }^{20}$ Generation of HBMEC cell lines was previously described. ${ }^{21}$ See the Online Supplementary Material and Methods for details.

\section{Physiological flow assays}

Physiological flow experiments were performed as previously described. ${ }^{22}$ See the Online Supplementary Material and Methods for details.

\section{Podosome formation assays}

Human dendritic cells (DC) were generated as described previously. ${ }^{23}$ See the Online Supplementary Material and Methods for details.

\section{VE-cadherin internalization assays \\ See the Online Supplementary Material and Methods for details.}

\section{Immunoprecipitation and Western blot analysis}

Immunoprecipitation and Western blotting were performed as previously described. ${ }^{24}$ See the Online Supplementary Material and Methods for further details.

\section{Statistics}

See the Online Supplementary Material and Methods for details.

\section{Results}

\section{BM vasculature is highly permeable for small molecules}

To test the vascular barrier in the BM during homeostasis, fluorescently-labeled $10 \mathrm{kDa}$ dextrans were intravenously administered to mice together with the vascular marker GS-I and allowed to circulate for 5 minutes, after which the mice were sacrificed (Figure 1A). To define vascular permeability, we employed whole mount multiphoton imaging of several organs and measured the fluorescent intensity of the dextrans in the vascular tissue microenvironment within a perimeter of one cell layer $(8 \mu \mathrm{m})$ around individual blood vessels (Figure 1B). To correct for potential loss of intensity at greater tissue depth, fluorescence intensity of the dextrans was normalized to intensity values of the GS-I vascular staining (Figure 1C). We determined the vascular permeability of BM, liver, lung and heart as a ratio to the vascular permeability of the brain, where vascular permeability is exceptionally low (Figure 1D-E). Vascular permeability in the BM was comparable to that of the liver, and approximately 2-3 times higher than that of the lung and heart, respectively (Figure $1 \mathrm{D}, 1 \mathrm{E})$. Thus, BM vessels are permeable for small dex- 
A

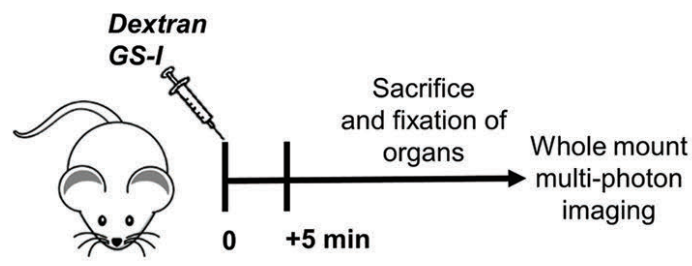

C

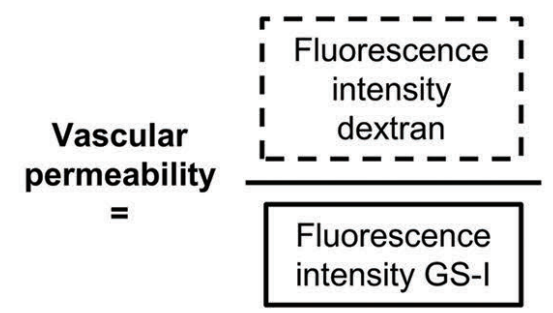

B
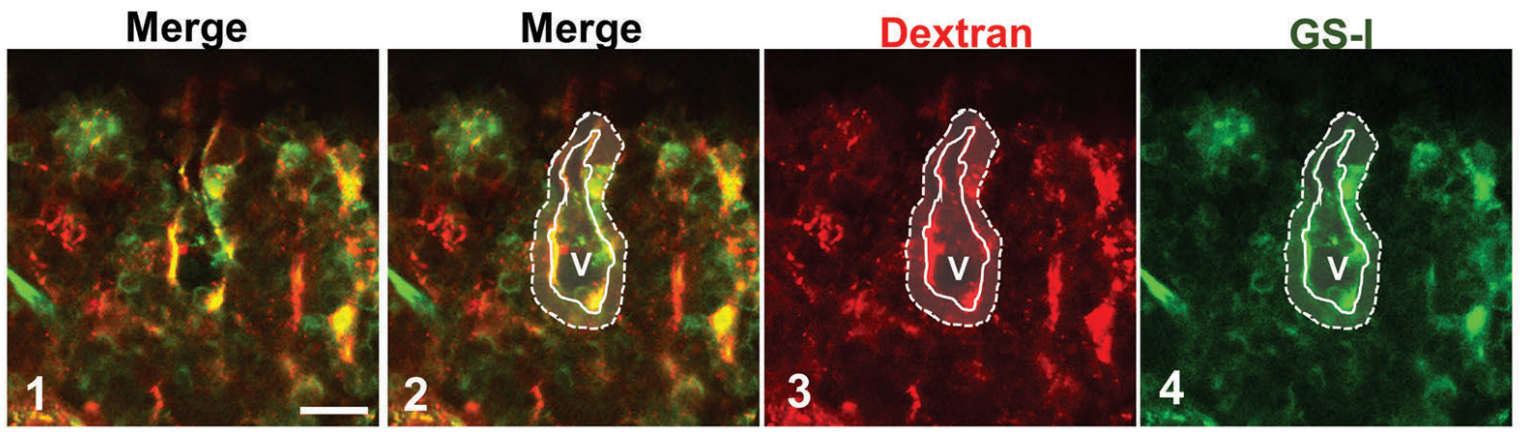

D
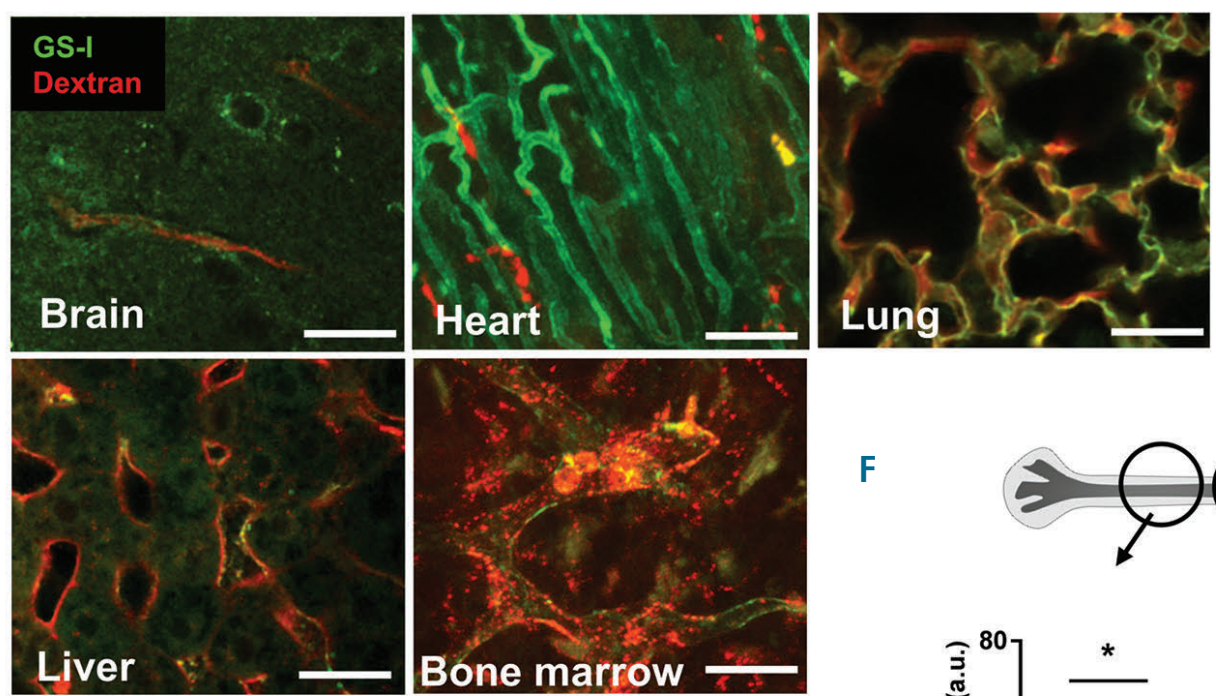

$\mathrm{F}$
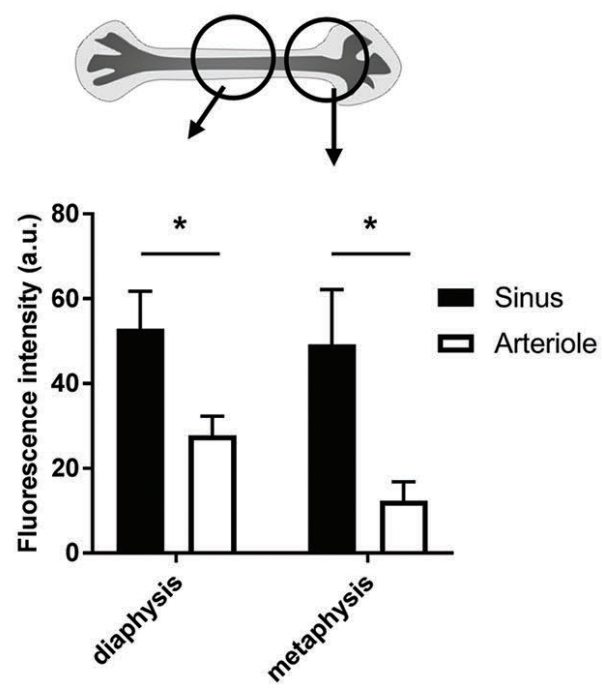

E

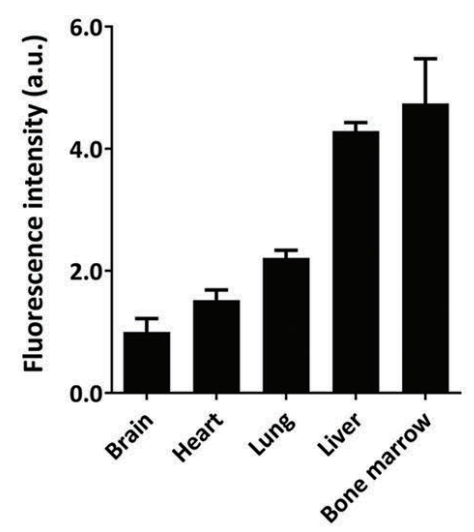

Figure 1. Bone marrow vasculature is highly permeable for small molecules. (A) Schematic summary of experimental set up. (B) Bone marrow (BM) imaging after injection of fluorescent dyes with in green GS-I for vessel labeling and in red $10 \mathrm{kDa}$ dextran (panel 1). Panel 2-4 show identification of a vessel (solid line) with red fluorescence detection outside of the vessel (dotted line), as a measurement of vascular permeability. $V$ indicates the vessel lumen. (C) Mathematical equation for vascular permeability: Fluorescence intensity of dextran (solid line in 1B) is divided by the fluorescence intensity of the vessel stain in the selected area (dotted line in 1B). (D) Detection of vascular permeability in several organs as indicated. (E) Quantification of the vascular permeability per organ, calculated as described in (C) $(n=2)$. (F) Detailed analysis of vascular permeability in specific BM regions: Diaphysis and metaphysis and discrimination between arterioles and sinusoids, based on intensity of GS-I stainings $(n=5)$. Scale bars: $25 \mu \mathrm{m}$. 
trans, similar to fenestrated sinusoids of the liver.

Within the BM, we discriminated between sinusoids and arterioles in the metaphysis and diaphysis area, based on CD31 intensity staining, according to Bixel and coworkers. ${ }^{4}$ We found that basal permeability in sinusoids was significantly higher than in arterioles (Figure 1F). These data indicate that baseline permeability differs per type of vessel in the BM.

\section{VE-cadherin regulates BM vascular permeability in homeostatic conditions and after irradiation}

As VE-cadherin is recognized as the major regulator of vascular permeability, ${ }^{25}$ we investigated whether vascular permeability in the BM is also regulated by VE-cadherin. To block homotypic binding of VE-cadherin at the endothelial junctions, we injected mice intravenously with a blocking antibody against VE-cadherin (clone 7519) 4 hours prior to the injection of fluorescently labeled 10 $\mathrm{kD}$ dextran (Figure $2 \mathrm{~A}$ ). It has previously been shown that VE-cadherin-blocking antibodies increased vascular permeability of the heart, lung and lymph nodes in vivo, ${ }^{19,26}$ but less is known about their effect on the integrity of the $\mathrm{BM}$ vasculature. We found that loss of VE-cadherin function resulted in increased vascular permeability in both sinusoids and arterioles in the BM (Figure 2B-C) compared to non-treated conditions. Blocking VE-cadherin resulted in increased permeability in the heart as well (Online Supplementary Figure S1A). Interestingly, the effects of VEcadherin blockage in the BM on the permeability of 500 $\mathrm{kDa}$ dextran did not result in a significant increase (Figure 2B-C and Online Supplementary Figure S1A), indicating that the blockage does not massively disrupt endothelial junctions. When locking VE-cadherin-based junctions, using the VE-cadherin-alpha-catenin chimera knock-in animals, we found that under homeostatic conditions, the BM vascular permeability, measured with $10 \mathrm{kDa}$ dextrans, was significantly reduced compared to WT controls (Figure $2 \mathrm{D}-\mathrm{E})$. These data indicate that VE-cadherin regulates vascular permeability during homeostasis in a paracellular fashion.

From a clinical perspective, total body irradiation is commonly applied before hematopoietic stem cell transplantation (HSCT) to enable homing of sufficient numbers of HSPC. ${ }^{27}$ We measured vascular permeability of BM sinusoids of low dose irradiated mice with $10 \mathrm{kDa}$ dextrans (Figure 3A) and found that vascular permeability in the $B M$ is significantly increased after irradiation (Figure $3 B-C)$, in line with previous studies. ${ }^{28-30}$ To substantiate the role of VE-cadherin in regulating the integrity of the BM vasculature, we examined permeability in VE-cadherin- $\alpha$ catenin chimera mice upon low dose irradiation. The effect of stabilizing endothelial junctions on BM vascular permeability was still apparent after low dose irradiation of VE- $\alpha$-catenin fusion mice, as BM vascular permeability was significantly lower compared to irradiated controls, in sinusoids as well as in arterioles (Figure 3D-E). Taken together, these data show that VE-cadherin is important in regulating vascular permeability in the BM. Using the VEcadherin-GFP transgenic knock-in mouse model, we did not observe clear changes in VE-cadherin distribution in the BM after low-dose irradiation, suggesting that the physical structure of the BM vasculature is not affected by low dose irradiation (Figure 3F). Also VE-cadherin expression was unaltered, as determined by Western blotting (data not shown). VE-cadherin function is known to be regulated by phosphorylation. ${ }^{31,32}$ Consequently, VE-cadherin is internalized. To study in more detail if irradiation alters VE-cadherin internalization or phosphorylation levels that undermine the endothelial cell-cell junction integrity, we used in vitro human umbilical vein endothelial cells (HUVEC) and found that low-dose irradiation promoted internalization of VE-cadherin (Online Supplementary Figure $S 1 B-D$ ). However, no clear increase in tyrosine phosphorylation of VE-cadherin or a loss of the interaction of $\mathrm{p} 120$-catenin with VE-cadherin was observed, although both events are known to be involved in VE-cadherin internalization ${ }^{31,33}$ (Online Supplementary Figure S1E-F). Although serine phosphorylation may also be involved in VE-cadherin internalization, ${ }^{32}$ we did not study this. From these data, we concluded that low-dose irradiation does not affect the overall physical structure of endothelial junctions in the $\mathrm{BM}$ vasculature, but rather induces a change in the regulation of vascular permeability, possibly by increasing the internalization of VE-cadherin.

\section{Loss of VE-cadherin function increases HSPC homing to the BM}

We next examined whether VE-cadherin also regulates homing of HSPC to the BM. C-kit HSPC were adoptively transferred into low dose-irradiated mice in the presence or absence of a blocking VE-cadherin antibody (Figure 4A). After 16 hours, mice were sacrificed and the presence of donor HSPC in the BM, lung, spleen, and liver was determined (Figure $4 \mathrm{~B}$ ). We found that in the mice in which VE-cadherin function was blocked, homing of lineage-Sca- $1^{+} \mathrm{c}-\mathrm{kit}^{+}$(LSK) cells to the BM was significantly increased 2-fold, compared to controls (Figure 4C). There was also a tendency towards increased homing of donor HSPC to spleen in anti-VE-cadherin-injected mice albeit not significant (Figure 4C). Homing of HSPC to the lung and liver was not affected by anti-VE-cadherin antibodies. The reason for this may be that percentages of donor HSPC in other organs than the BM were $>40$ fold lower than in the BM (data not shown), implying that the presence of adoptively transferred HSC in these organs was not due to directed migration. This is not surprising considering that CXCL12, the most important chemokine for HSPC, is expressed in the BM at much higher levels than in any other organ. ${ }^{34}$ To study if increased homing also resulted in increased engraftment, we measured the blood content after 1, 2 and 3 months of blocking of VE-cadherin function, as described above. The results showed a significant increase in myeloid cell numbers in the blood after 3 months (Online Supplementary Figure S2A). These data were supported by the BM cell phenotyping after 3 months (Online Supplementary Figure S2B). Also, here we found the myeloid cell fraction to be significantly increased, whereas the other leukocyte types, including the LSK cells were not (Online Supplementary Figure S2B). Shimoto and colleagues recently reported that under nonirradiation conditions, large numbers of HSPC need to be transplanted to reach normal homing of stem cells to the BM. $^{35}$ Additionally, homing is improved without total body irradiation preconditioning. ${ }^{36}$ To test if opening the endothelial junctions by blocking VE-cadherin may promote this, we performed this experiment. However, the results showed that also under non-irradiated conditions, no increase in engraftment upon treatment with the VEcadherin antibody was observed (Online Supplementary 
Figure S2C). Together, these data show that blocking VEcadherin promotes initial homing of HSPC but does not have a long-term effect on engraftment.

As we observed a clear correlation between increased BM vascular permeability and increased homing of HSPC to the BM upon loss of VE-cadherin function, we expected decreased homing of HSPC to the BM of VE-cadherin- $\alpha$ catenin fusion mice that have decreased BM vascular permeability. Surprisingly, we did not observe decreased homing of HSPC to the BM of VE-cadherin- $\alpha$-catenin fusion mice compared to WT controls (Figure 4D), indicating that permeability and transmigration in the $\mathrm{BM}$ are

A

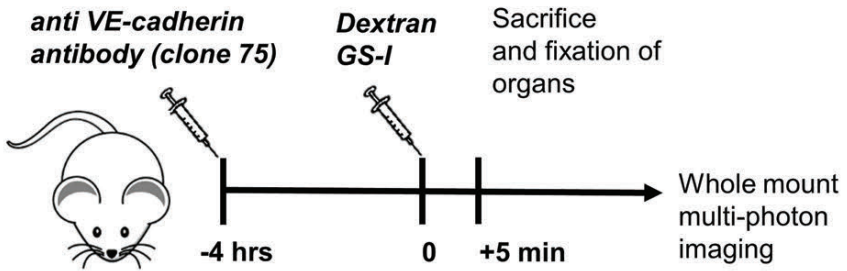

B
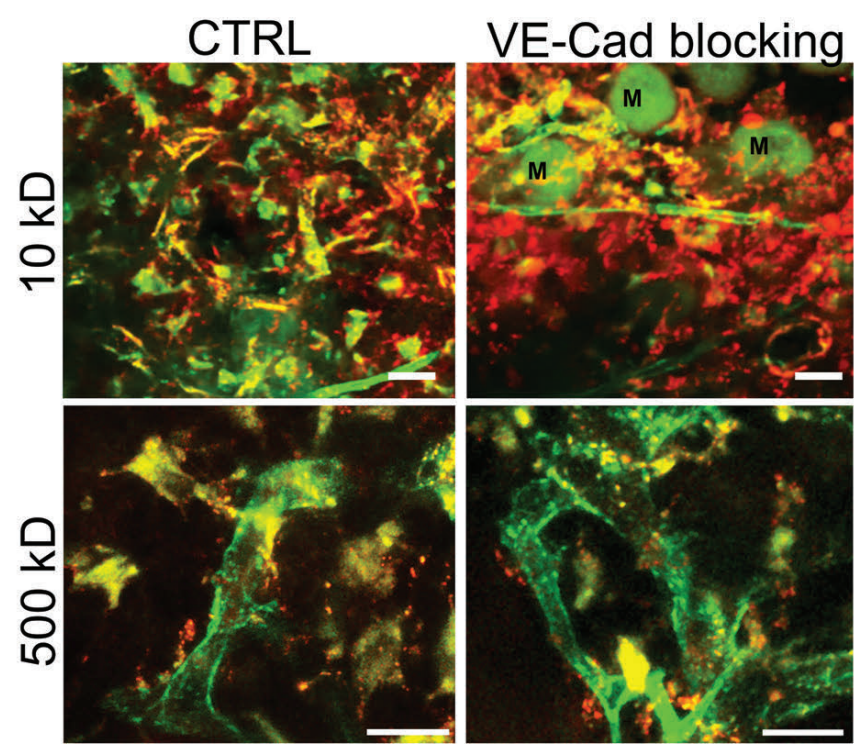

D

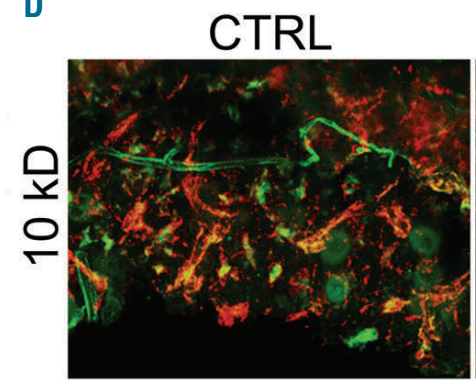

C
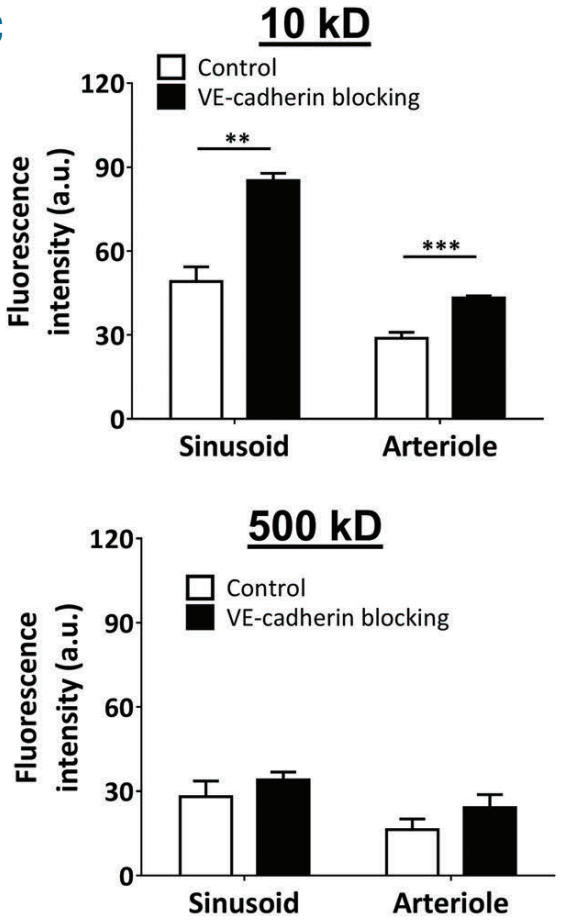

E

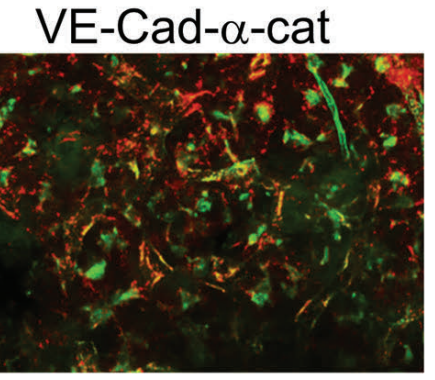

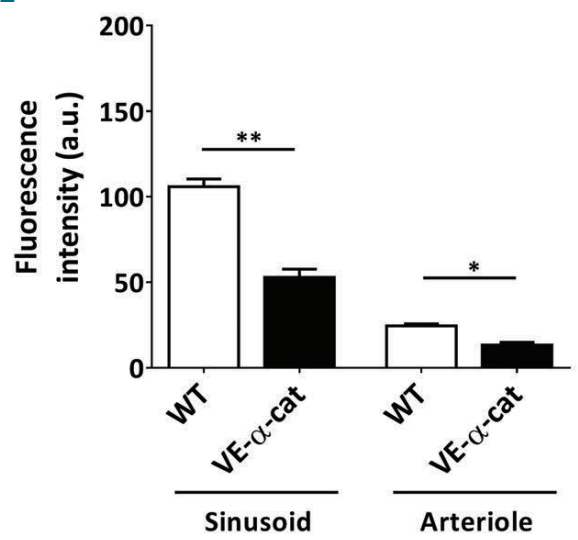

Figure 2 Anti-VE-cadherin antibodies increase bone marrow vascular permeability in homeostatic conditions and after irradiation. (A) Schematic summary of experimental set up. (B) Bone marrow (BM) imaging after injection of fluorescent dyes with in green GS-I for vessel labeling and in red 10 and 500 kDa dextran as indicated. M: megakaryocytes. (C) Quantification of vascular permeability in arterioles and sinusoids in metaphysis and diaphysis after blocking VE-cadherin (10 kD: $n=7-8$ per group, $500 \mathrm{kD}: \mathrm{n}=4$ per group). (D) BM imaging of wild-type and VE-cadherin- $\alpha$-catenin chimera mice after injection of fluorescent dyes with in green GSI for vessel labeling and in red $10 \mathrm{kDa}$ dextran ( $\mathrm{n}=3-4$ per group). (E) Quantification of vascular permeability in arterioles and sinusoids in wild-type and VE-cadherin$\alpha$-catenin chimera mice as indicated. Scale bars: $25 \mu \mathrm{m}$, ctrl: control. 
regulated separately, in line with our previous finding where we showed that the endothelium actively limits vascular permeability during neutrophil extravasation. ${ }^{37}$ There was also no significant difference in HSPC homing to the spleen and liver in VE- $\alpha$-catenin fusion mice, although a trend for more homing to the lungs was detected (Figure 4D). To verify that the donor HSPC which we identified in the BM of VE- $\alpha$-catenin fusion mice by fluorescence activated cell sorting (FACS) analysis were not trapped in the vascular bed of the BM, cryosections of the BM of transplanted WT and VE-cadherin- $\alpha$-catenin fusion mice were analyzed (Online Supplementary Figure S2D). Here, we found that the majority of adoptively transferred
HSPC were located inside the BM parenchyma (Online Supplementary Figure S2E). Moreover, the antibody effect was gone after 16 hours of treatment, based on an increase in BM vascular permeability (Online Supplementary Figure $S 2 D, F)$. These data showed that stabilizing endothelial junctions does not impair the homing of HSPC to the BM.

HSPC predominantly use the transcellular route of migration over BM endothelium and form podosome-like structures

Our finding that homing of HPSC in VE-cadherin- $\alpha$ catenin fusion mice is unaltered suggested that these cells crossed the BM endothelium in a transcellular manner,

A

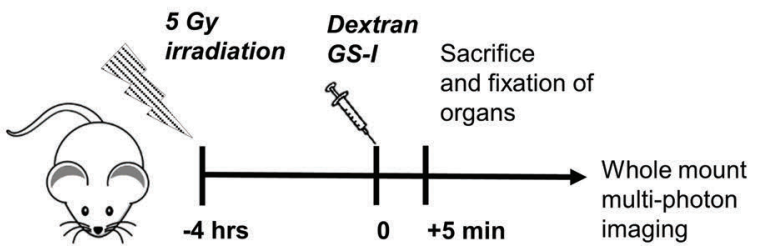

B

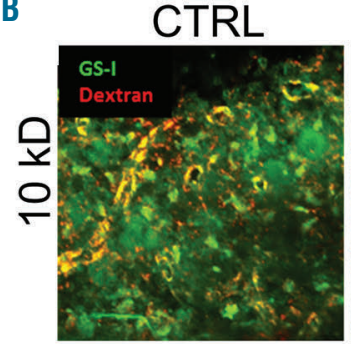

D
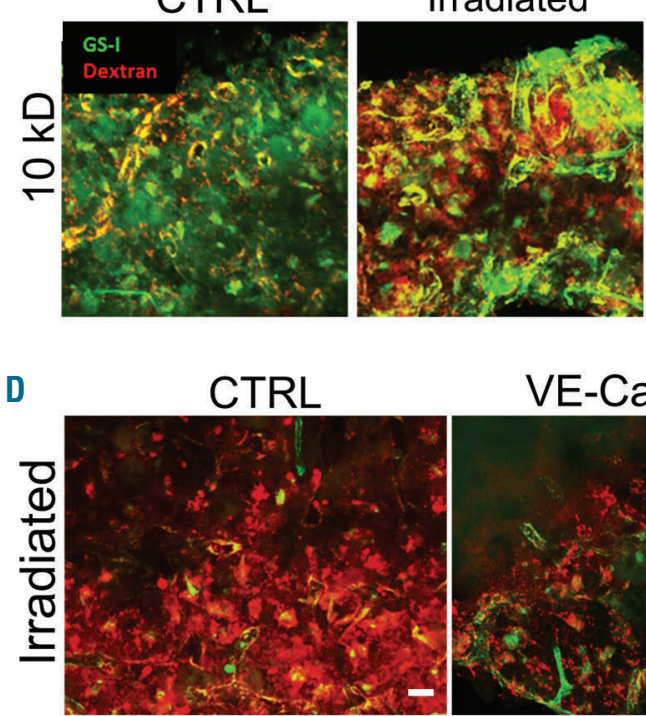

Irradiated

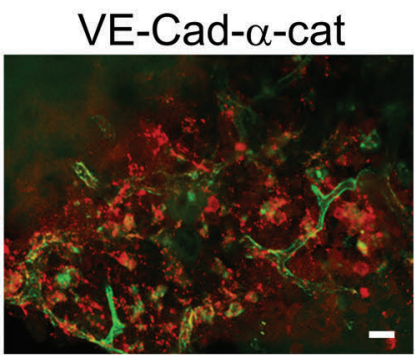

$\mathrm{F}$

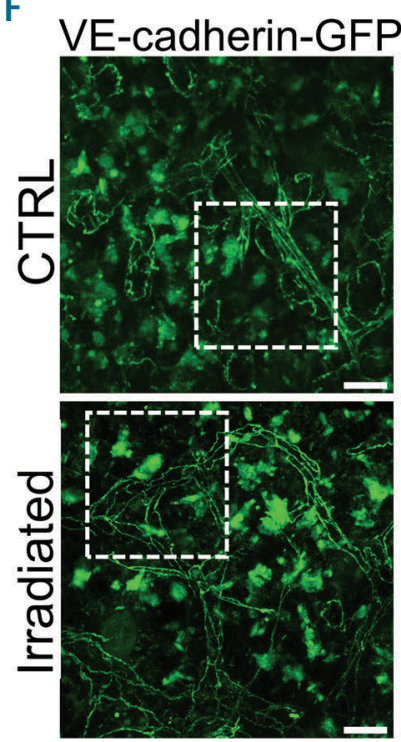

Irradiated +
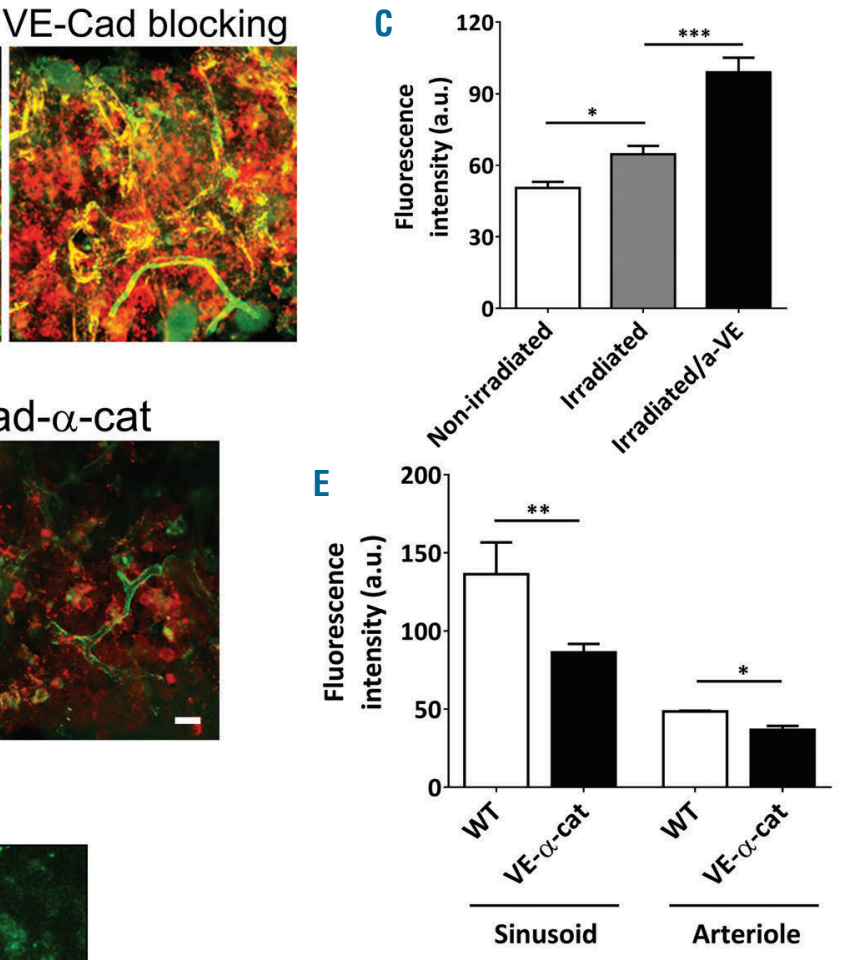

Figure 3. Reduced bone marrow vascular permeability in VE-cadherin$\alpha$-catenin fusion mice. (A) Schematic summary of experimental set up. (B) Bone marrow (BM) imaging of wild-type, VE-cadherin- $\alpha$-catenin chimera mice and supplemented with anti-VE-cadherin antibody after injection of fluorescent dyes with in green GS-I for vessel labeling and in red $10 \mathrm{kDa}$ dextran ( $n=3-4$ per group). (C) Quantification of vascular permeability in sinusoids in wild-type, VE-cadherin- $\alpha$-catenin chimera mice and supplemented with anti-VE-cad antibody as indicated. (D) BM imaging of wild-type and VE-cadherin- $\alpha$-catenin chimera mice after 5 Gy irradiation after injection of fluorescent dyes with in green GS-I for vessel labeling and in red $10 \mathrm{kDa}$ dextran ( $n=3-4$ per group). (E) Quantification of vascular permeability in arterioles and sinusoids in wild-type and VE-cadherin- $\alpha$-catenin chimera mice upon irradiation as indicated. (F) BM vasculature using a VE-cadherin-GFP knock-in mice showed proper VE-cadherin lining in control and upon irradiation conditions. Images on right are zoom from dotted box. Scale bars: $25 \mu \mathrm{m}$; ctrl: control. 
rather than through the cell-cell junctions. As it is technically challenging to discriminate between both routes using in vivo imaging of the BM vasculature, we employed an in vitro approach, where we used human $\mathrm{CD} 34^{+} \mathrm{CB}$ HSPC and immortalized human BM endothelial cells (HBMEC). ${ }^{21}$ To properly discriminate between the para- or transcellular route, HBMEC were transfected with LifeactGFP to visualize F-actin, activated for 4 hours with IL-1 $\beta^{21}$ and 30 minutes prior to adding the $\mathrm{CD} 34^{+}$cells, HBMEC were incubated with fluorescently-labeled, non-blocking VE-cadherin antibodies to visualize endothelial junctions (Figure 5A). ${ }^{22}$ Interestingly, we found that the vast majority $(\sim 75 \%)$ of HSPC crossed the HBMEC transcellularly (Figure 5B). Pretreatment of the endothelial cells with a blocking VE-cadherin antibody promoted paracellular migration, indicating that these cells can cross through the junctions (Figure 5B). Although it is not fully understood how the transcellular migration route is regulated, it is believed that membrane fusion events are required to form a so-called transcellular pore. ${ }^{38}$ Therefore, we blocked membrane fusion activity in endothelial cells using the SNARE-complex inhibitor $\mathrm{N}$-Ethylmaleimide (NEM) ${ }^{38}$ HSPC migration across a confluent HBMEC layer that was treated with NEM showed a significant inhibition of the number of HSPC transmigrating towards CXCL12 (Figure 5C). These data indicated that the formation of transcellular migration pores is the major route for HSPC to cross HBMEC. It should be

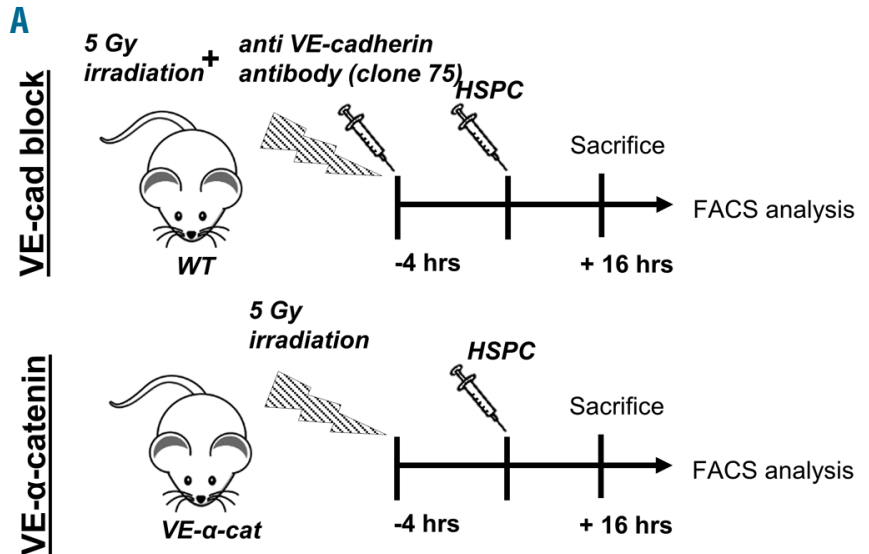

C
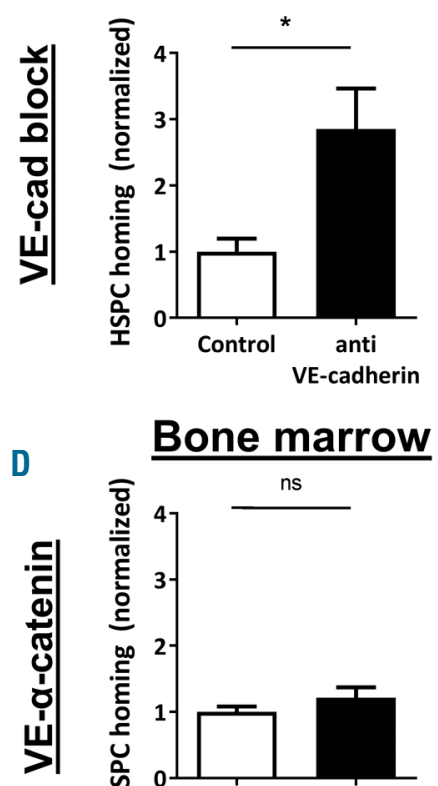
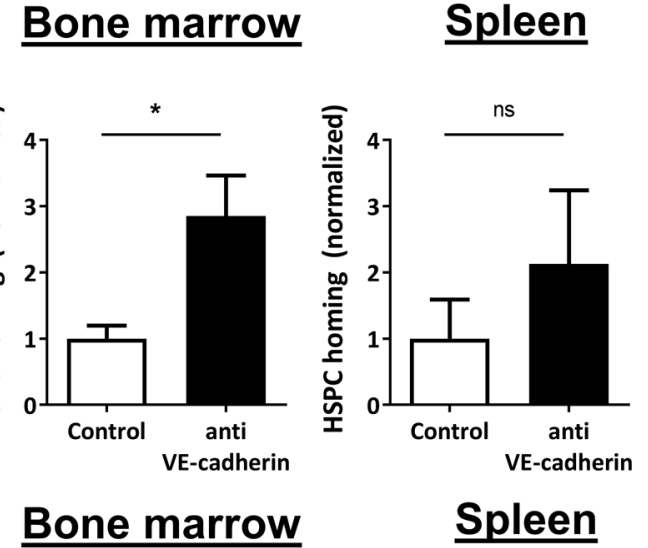

Spleen

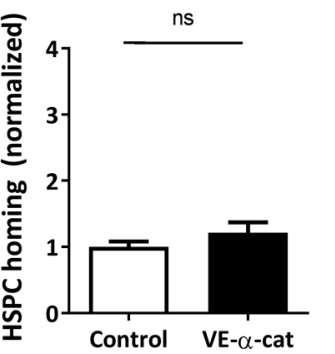

B

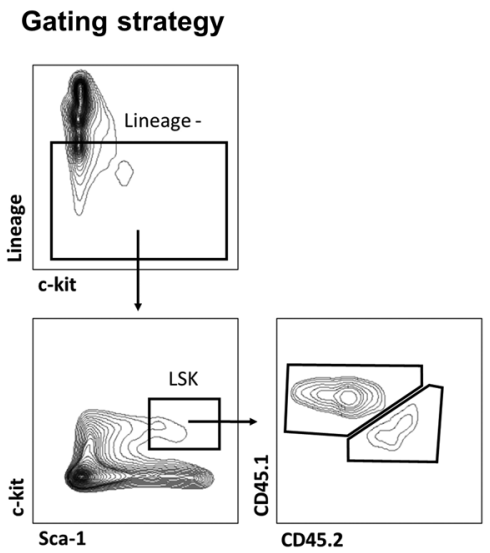

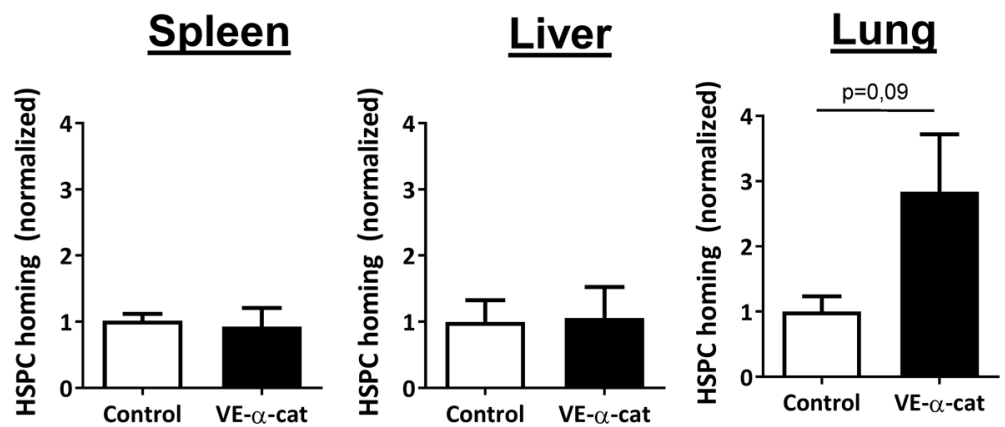

Figure 4. Anti-VE-cadherin antibodies increase hematopoietic stem and progenitor cells homing to the bone marrow. (A) Schematic summary of experimental set up for two groups: anti-VE-cadherin antibody administration and VE-cadherin/alpha-catenin chimera (VE- $\alpha$-cat). Experiment also includes control group. (B) Gating strategy to select for LSK cells (murine hematopoietic stem and progenitor cells [HSPC]) using flow cytometry. (C) Normalized homing efficiency of HSPC to different organs after 16 hours (hrs) of transplantation in control and anti-VE-cadherin antibody group ( $n=4-5$ per group, representative of two independent experiments). (D) Normalized homing efficiency of HSPC to different organs after 16 hrs of transplantation in wild-type and VE- $\alpha$-cat groups ( $n=4-5$ per group). 
A

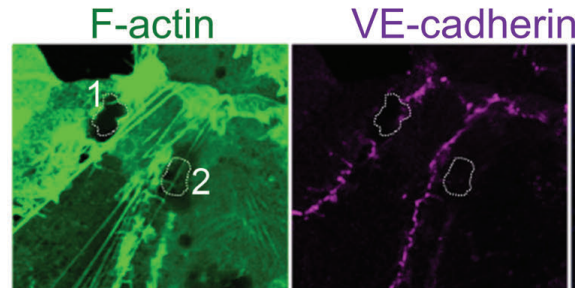

Position HSPC 1
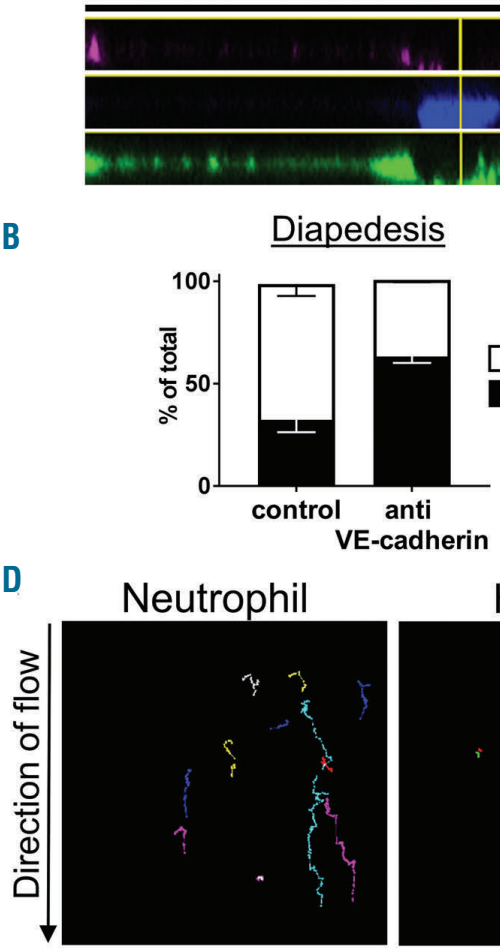

E

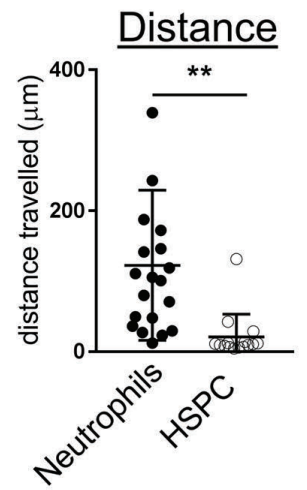

\section{G}

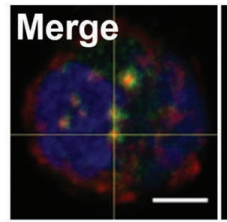

XZ projection

H

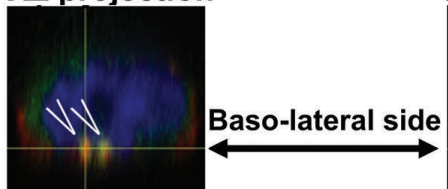

HSPC
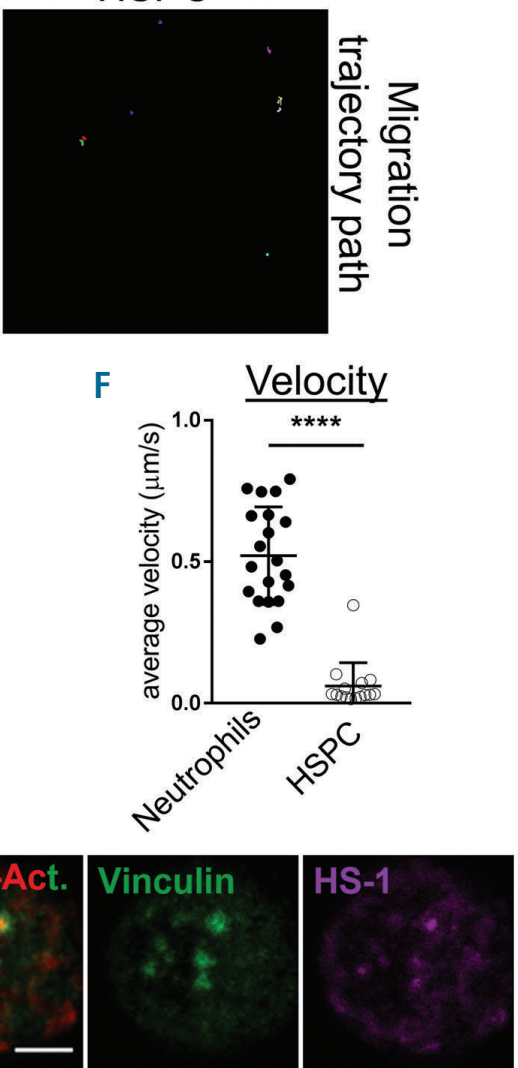

XZ projection
C

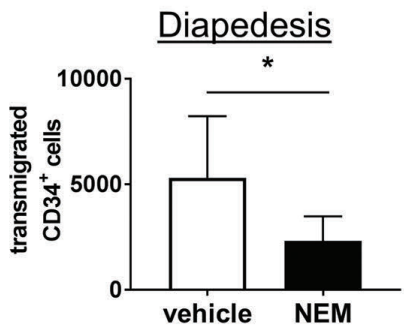

Figure 5. Hematopoietic stem and progenitor cells predominantly use the transcellular route of migration across bone marrow endothelium by forming podosome-like structures. (A) IL1 $\beta$-pretreated immortalized human BM endothelial cells (HBMEC) were cultured and transfected with GFP-LifeAct (green) to monitor F-actin dynamics during hematopoietic stem and progenitor cells (HSPC) interactions. Both migration routes were detected for HSPC migration across HBMEC: paracellular with junctions marked by VE-cadherin (magenta), and transcellular, nuclei stained in blue and DIC shows overview. White dotted lines indicate localization of human HSPCs with \#1 representing paracellular migration and \#2 representing transcellular migration. Lower panel shows XZ projection with position HSPC 1 for paracellular migration (illustrated by the two magenta stains at the side) and position HSPC 2 for transcellular migration. Scale bar: 20 um. (B) Quantification of transmigration route of HSPC. Open bar represents percentage of cells that cross transcellularly, and closed bars represent percentage that crosses paracellularly (mean \pm standard error of the mean [SD], $\mathrm{n}=3$ per group). Anti-VE-cadherin indicates that VE-cadherin function is blocked and promotes paracellular pathway. (C) Blocking of membrane fusion events in HBMEC by $\mathrm{N}$-Ethylmaleimide (NEM) impairs HSPC transendothelial migration towards CXCL12 in an in vitro Transwell system (Mean \pm SD, $\mathrm{n}=3$ per group). (D) Migration tracks of cells under flow conditions show highly motile neutrophils and passive HSPC, quantified by distance traveled on the apical surface of HBMEC in $\mu \mathrm{m}$ $(E)$ or average velocity $(\mu \mathrm{m} / \mathrm{s})(\mathrm{F})$. These experiments were independently carried out at least three times. (G) HSPC were treated for 30 minutes with PMA showing the induction of podosomes, based on typical podosome markers: vinculin (green) in the circle and HS-1 (magenta) and F-actin (red) in the core. Scale bar: $3 \mu \mathrm{m}$. (H) Orthogonal projection shows the distribution of the podosomes at the basolateral surface (open arrowheads) with F-actin in red, vinculin in green and the nucleus in blue. 
noted that NEM treatment did also reduce the endothelial cell monolayer barrier.

The fact that HPSC prefer the transcellular route is a surprising finding, considering that most immune cells use the paracellular route for transmigration. ${ }^{39}$ Although the exact mechanism of diapedesis is still under debate, transand paracellular migration are most likely regulated by different molecular mechanisms. On the endothelial side, VE-cadherin plays a key role in paracellular migration but not in transcellular migration. ${ }^{40}$ From the leukocyte, Mac$1 / C D 11 b$ is thought to be essential for crawling towards the nearest endothelial junction, thus facilitating paracellular migration. ${ }^{41}$ Interestingly, only a small subset of HSPC expresses Mac-1/CD11b,42 suggesting that the majority of HSPC is incapable to efficiently migrate to the nearest endothelial junctions. To test this hypothesis, we used in vitro physiological flow conditions and live-cell real-time imaging ${ }^{22}$ to quantify HSPC crawling over HBMEC monolayers. HSPC did not crawl on HBMEC monolayers, whereas neutrophils did (Figure 5C). Quantification of the migration tracks showed that both the distance and velocity of HSPC was drastically reduced compared to neutrophils (Figure 5D-E). Off note, neutrophils crawl equally well on HUVEC as on HBMEC monolayers (Online Supplementary Figure S3A-C). Thus, our data suggest that HPSC are not equipped to crawl on the endothelium to locate the nearest endothelial junction, but rather start their transmigration event immediately upon adhesion, potentially in a transcellular manner.

As of yet, nothing is known on how HSPC may initiate transcellular migration. ${ }^{43}$ For transcellular migration of T-lymphocytes, it has been suggested that these cells make invasive protrusions, or so-called 'podosomes' to palpate the surface of the endothelium and initiate transcellular pore formation. ${ }^{38}$ Other cellular projections such as uropod and filopodia have been observed in HPSC, ${ }^{44}$ but the formation of podosomes by HSPC has not yet been documented. Podosomes consist of a protrusive actin core and an adhesive ring of integrins and actinadaptor proteins, such as vinculin and cortactin, or its hematopoietic homologue, hematopoietic lineage cellspecific protein 1 (HS-1). ${ }^{45,46}$ To determine whether HSPC can form podosomes, we allowed HSPC to adhere to fibronectin-coated coverslips and exposed them to phorbol 12-myristate 13-acetate (PMA) for 30 minutes. As a positive control we induced podosome formation in cultured dendritic cells, where we indeed observed clusters of podosomes with a ring of vinculin and a core containing actin and HS-1, as previously described ${ }^{46}$ (Online Supplementary Figure S3D-E). Remarkably, we could identify similar, podosome-like structures in human HPSC (Figure 5G-H), indicating that HSPC are equipped with the machinery to induce podosomes that may allow them to migrate through endothelial cells in a transcellular manner. Additional experiments were added to show that murine LSK cells can also induce podosomes as well (Online Supplementary Figure S3F).

\section{Discussion}

We show that VE-cadherin regulates the vascular integrity of the BM vasculature and inhibition of VE-cadherin function can promotes the homing of HPSC, although this has no consequence for the engraftment potential. Moreover, we show that human as well as murine HSPC cross the BM endothelium in a transcellular manner under homeostatic conditions, and can initiate podosome-like structures that may assist these cells on their way through. Our in vivo data suggest that murine HSPC cross the vasculature in a transcellular manner. Previous observations using electron microscopy already indicated that leukocytes crossed the vasculature of the BM in a transcellular manner. ${ }^{12,13}$ The fact that HSPC do not crawl on endothelial monolayers, presumably because they predominantly express LFA-1, known to regulate firm adhesion, and not so much Mac-1, required for directional migration, ${ }^{47}$ also points towards the effective use of the transcellular pathway. It remains unclear why HSPC prefer the trans- over the paracellular route. Interestingly, our data furthermore show that the route to cross the endothelium is not exclusively regulated. When opening cell-cell junctions, by blocking VE-cadherin interactions, and thereby increasing vascular permeability, more HSPC cross the endothelium. These data show that junctional resistance also plays a role in the molecular decision for HSPC transmigration. Putatively, transcellular migration of HSPC continues upon administration of a VE-cadherin antibody, and the observed increase in HSPC homing to the BM can be explained by an increase of paracellular migration of HSPC. The fact that HSPC predominantly use the transcellular route to cross the endothelium may also explain that HSPC homing to the BM is not impaired in VE-cadherin- $\alpha$-catenin fusion mice. It was previously shown that, in contrast to our data on HSPC homing, neutrophil migration to inflamed cremaster, lung, and skin is significantly impaired in VE-cadherin- $\alpha$-catenin fusion mice. However, this is not surprising considering that neutrophils almost exclusively use the paracellular route of transmigration. ${ }^{37,39,41,48}$ Interestingly, also in these studies, it was shown that lymphocyte homing was not affected in the VE-cadherin- $\alpha$-catenin fusion mice?

Although we have shown that anti-VE-cadherin antibodies increase the homing of HSPC to the BM, we did not measure an increase in the engraftment. Together with the notion that HSPC cross the endothelium in a transcellular fashion, our data indicate that VE-cadherin is not the preferred target to increase HSPC transplantations after irradiation. Rather, a target for transcellular migration would be more beneficial. Unfortunately, until today, it is not understood how leukocytes migrate through the endothelial cell body. One potential mechanism may be the induction of podosomes. Podosomes have been described for DC and are believed to degrade the extracellular matrix. ${ }^{49}$ For $\mathrm{T}$ lymphocytes, it is recognized that podosomes may help these cells to cross in a transcellular manner. ${ }^{5,50}$ The fact that $T$ cells and HSPC show the same transmigration kinetics, i.e., they firmly adhere to the initial point of attachment and stabilize, indicate that they may use the same molecular mechanisms to cross the endothelium. Indeed, blocking endothelial vesicle transport using NEM in both cases reduces the number of cells that transmigrates. Thus, we put forward that HSPC, like $\mathrm{T}$ lymphocytes, use podosomes to cross the vascular endothelium in a transcellular manner.

Taken together, we report that although HSPC predominantly use the transcellular route for transendothelial migration in homeostasis, blocking VE-cadherin homotypic interactions favors paracellular migration of HSPC and increases homing of HSPC into the BM. This does not 
result in increased long-term engraftment. Our work offers valuable insight into the mechanisms of HSPC migration across the BM endothelium.

\section{Acknowledgments}

The authors thank Simon Tol, Erik Mul, and Aafke de Ligt for technical assistance and the staff of the animal facilities of the NKI and the Max Planck Institute for Molecular Biomedicine for excellent animal care. The authors thank Alessandra Cambi and Koen van den Dries for sharing their expertise on podosomes. $A G P$ received a pre-doctoral scholarship from the Mexican
National Council for Science and Technology (Conacyt, 369767); and a travel fellowship grant from the Journal of Cell Science, The Company of Biologists Limited (WWW. biologists.com) to finance a research stay in the laboratory of $J D v B$. Work in the laboratory of $M S$ is funded by Conacyt (grant 233395).

\section{Funding}

This work was supported by Sanquin Research (PPOC13$030 P$ grant). The authors declare no competing financial interests.

\section{References}

1. Dercksen MW, Rodenhuis S, Dirkson MK, et al. Subsets of CD34+ cells and rapid hematopoietic recovery after peripheralblood stem-cell transplantation. J Clin Oncol. 1995;13(8):1922-1932

2. Voermans C, van Hennik PB, van der Schoot CE. Homing of human hematopoietic stem and progenitor cells: new insights, new challenges? J Hematother Stem Cell Res. 2001:10(6):725-738.

3. Lapidot T, Dar A, Kollet O. How do stem cells find their way home? Blood. 2005;106(6):1901-1910.

4. Carman CV. Mechanisms for transcellular diapedesis: probing and pathfinding by 'invadosome-like protrusions'. J Cell Sci. 2009;122(17):3025-3035.

5. Carman CV, Sage PT, Sciuto TE, de la Fuente $\mathrm{MA}$, et al. Transcellular diapedesis is initiated by invasive podosomes. Immunity. 2007; 26(6):784-797.

6. Martinelli R, Zeiger AS, Whitfield M, et al. Probing the biomechanical contribution of the endothelium to lymphocyte migration: diapedesis by the path of least resistance. J Cell Sci. 2014;127(Pt 17):3720-3734

7. Schulte D, Kuppers V, Dartsch N, et al. Stabilizing the VE-cadherin-catenin complex blocks leukocyte extravasation and vascular permeability. EMBO J. 2011;30(20):41574170.

8. Yang L, Froio RM, Sciuto TE, Dvorak AM, Alon R, Luscinskas FW. ICAM-1 regulates neutrophil adhesion and transcellular migration of TNF-alpha-activated vascular endothelium under flow. Blood. 2005; 106(2):584-592.

9. Abadier M, Haghayegh JN, Cardoso AL, et al. Cell surface levels of endothelial ICAM1 influence the transcellular or paracellular T-cell diapedesis across the blood-brain barrier. Eur J Immunol. 2015;45(4):10431058.

10. Millan J, Hewlett L, Glyn M, Toomre D, Clark P, Ridley AJ. Lymphocyte transcellular migration occurs through recruitment of endothelial ICAM-1 to caveola- and F-actinrich domains. Nat Cell Biol. 2006;8(2):113123.

11. Vestweber D, Broermann A, Schulte D. Control of endothelial barrier function by regulating vascular endothelial-cadherin. Curr Opin Hematol. 2010;17(3):230-236

12. De Bruyn PP, Michelson S, Thomas TB. The migration of blood cells of the bone marrow through the sinusoidal wall. J Morphol. 1971;133(4):417-437.

13. Muto M. A scanning and transmission electron microscopic study on rat bone marrow sinuses and transmural migration of blood cells. Arch Histol Jpn. 1976;39(1):51-66.

14. Sarin H. Physiologic upper limits of pore size of different blood capillary types and another perspective on the dual pore theory of microvascular permeability. J Angiogenes Res. 2010;2:14.

15. Winderlich M, Keller L, Cagna G, et al. VEPTP controls blood vessel development by balancing Tie-2 activity. J Cell Biol. 2009;185(4):657-671.

16. Dartsch N, Schulte D, Hagerling R, Kiefer F, Vestweber D. Fusing VE-cadherin to alphacatenin impairs fetal liver hematopoiesis and lymph but not blood vessel formation. Mol Cell Biol. 2014;34(9):1634-1648.

17. Winderlich M, Keller L, Cagna G, et al. VEPTP controls blood vessel development by balancing Tie-2 activity. J Cell Biol. 2009; 185(4):657-671.

18. Halai K, Whiteford J, Ma B, Nourshargh $S$, Woodfin A. ICAM-2 facilitates lumina interactions between neutrophils and endothelial cells in vivo. J Cell Sci. 2014;127(Pt 3):620-629.

19. Gotsch U, Borges E, Bosse R, et al. VE-cadherin antibody accelerates neutrophil recruitment in vivo. J Cell Sci. 1997;110( Pt 5):583-588

20. Klamer SE, Kuijk CG, Hordijk PL, et al. BIGH3 modulates adhesion and migration of hematopoietic stem and progenitor cells. Cell Adh Migr. 2013;7(5):434-449.

21. Rood PM, Calafat J, von dem Borne AE, Gerritsen WR, van der Schoot CE. Immortalisation of human bone marrow endothelial cells: characterisation of new cell lines. Eur J Clin Invest. 2000;30(7):618629.

22. Kroon J, Daniel AE, Hoogenboezem M, van Buul JD. Real-time imaging of endothelial cell-cell junctions during neutrophil transmigration under physiological flow. J Vis Exp. 2014;(90):e51766.

23. de Vries IJ, Eggert AA, Scharenborg NM, et al. Phenotypical and functional characterization of clinical grade dendritic cells. Immunother. 2002;25(5):429-438.

24. Timmerman I, Heemskerk N, Kroon J, et al A local VE-cadherin and Trio-based signaling complex stabilizes endothelial junctions through Rac1. J Cell Sci. 2015;128(18):3514.

25. Giannotta M, Trani M, Dejana E. VE-cadherin and endothelial adherens junctions: active guardians of vascular integrity. Dev Cell. 2013;26(5):441-454.

26. Corada M, Mariotti M, Thurston G, et al Vascular endothelial-cadherin is an important determinant of microvascular integrity in vivo. Proc Natl Acad Sci U S A. 1999; 96(17):9815-9820

27. Storb R, Sandmaier BM. Nonmyeloablative allogeneic hematopoietic cell transplantation. Haematologica. 2016;101(5):521-530.

28. Daldrup-Link HE, Link TM, Rummeny EJ, et al. Assessing permeability alterations of the blood-bone marrow barrier due to total body irradiation: in vivo quantification with contrast enhanced magnetic resonance imaging. Bone Marrow Transplant. 2000; 25(1):71-78

29. Shirota T, Tavassoli M. Alterations of bone marrow sinus endothelium induced by ionizing irradiation: implications in the homing of intravenously transplanted marrow cells. Blood Cells. 1992;18(2):197-214.

30. Zhou BO, Ding L, Morrison SJ Hematopoietic stem and progenitor cells regulate the regeneration of their niche by secreting Angiopoietin-1. Elife. 2015; 4:e05521.

31. Wessel F, Winderlich M, Holm M, et al Leukocyte extravasation and vascular permeability are each controlled in vivo by different tyrosine residues of VE-cadherin. Nat Immunol. 2014;15(3):223-230.

32. Gavard J, Gutkind JS. VEGF controls endothelial-cell permeability by promoting the beta-arrestin-dependent endocytosis of VE-cadherin. Nat Cell Biol. 2006;8(11):1223 1234.

33. Xiao K, Allison DF, Buckley KM, et al. Cellular levels of p120 catenin function as a set point for cadherin expression levels in microvascular endothelial cells. J Cell Biol. 2003;163(3):535-545.

34. Uhlen M, Fagerberg L, Hallstrom BM, et al Proteomics. Tissue-based map of the human proteome. Science. 2015; 347(6220): 1260419.

35. Shimoto $M$, Sugiyama $T$, Nagasawa $T$. Numerous niches for hematopoietic stem cells remain empty during homeostasis. Blood. 2017;129(15):2124-2131.

36. Quesenberry PJ, Colvin G, Abedi M Perspective: fundamental and clinical concepts on stem cell homing and engraftment: a journey to niches and beyond. Exp Hematol. 2005;33(1):9-19.

37. Heemskerk N, Schimmel L, Oort C, et al. Factin-rich contractile endothelial pores prevent vascular leakage during leukocyte diapedesis through local RhoA signalling. Nat Commun. 2016;7:10493.

38. Carman CV, Sage PT, Sciuto TE, et al Transcellular diapedesis is initiated by invasive podosomes. Immunity. 2007;26(6):784 797.

39. Woodfin A, Voisin MB, Beyrau M, et al. The junctional adhesion molecule JAM-C regulates polarized transendothelial migration of neutrophils in vivo. Nat Immunol. 2011;12(8):761-769.

40. Vestweber D. How leukocytes cross the vascular endothelium. Nat Rev Immunol 2015;15(11):692-704.

41. Phillipson M, Heit B, Colarusso P, Liu L, Ballantyne CM, Kubes P. Intraluminal crawling of neutrophils to emigration sites: a molecularly distinct process from adhesion in the recruitment cascade. J Exp Med. 
2006:203(12):2569-2575

42. Dercksen MW, Gerritsen WR, Rodenhuis S, et al. Expression of adhesion molecules on CD34+ cells: CD34+ L-selectin+ cells predict a rapid platelet recovery after peripheral blood stem cell transplantation. Blood. 1995;85(11):3313-3319.

43. Magnon C, Frenette PS. Hematopoietic stem cell trafficking. StemBook [Internet]. Cambridge (MA): Harvard Stem Cell Institute; 2008

44. Dykstra B, Ramunas J, Kent D, et al. Highresolution video monitoring of hematopoietic stem cells cultured in single-cell arrays identifies new features of self-renewal. Proc
Natl Acad Sci U S A. 2006;103(21):81858190

45. Dehring DA, Clarke F, Ricart BG, et al Hematopoietic lineage cell-specific protein 1 functions in concert with the WiskottAldrich syndrome protein to promote podosome array organization and chemotaxis in dendritic cells. J Immunol. 2011;186(8):4805-4818.

46. van den Dries K, Schwartz SL, Byars J, et al Dual-color superresolution microscopy reveals nanoscale organization of mechanosensory podosomes. Mol Biol Cell. 2013;24(13):2112-2123.

47. Heit B, Colarusso P, Kubes P. Fundamentally different roles for LFA-1, Mac-1 and alpha4integrin in neutrophil chemotaxis. J Cell Sci. 2005;118(Pt 22):5205-5220.

48. Feng D, Nagy JA, Pyne K, Dvorak HF, Dvorak AM. Neutrophils emigrate from venules by a transendothelial cell pathway in response to FMLP. J Exp Med. 1998; 187(6):903-915.

49. van den Dries K, Bolomini-Vittori M, Cambi A. Spatiotemporal organization and mechanosensory function of podosomes. Cell Adh Migr. 2014;8(3):268-272.

50. Carman CV, Springer TA. Transcellular migration: cell-cell contacts get intimate. Curr Opin Cell Biol. 2008;20(5):533-540. 